\title{
A Single Sensor Hand Geometry and Palm Texture Fusion for Person Identification
}

\author{
M. P. Dale \\ E\&TC Dept., \\ MES's College of Engineering, \\ Pune
}

\author{
M. A. Joshi \\ E\&TC Dept \\ College of Engineering, Pune
}

\author{
H. J. Galiyawala \\ E\&TC Dept \\ College of Engineering, Pune
}

\begin{abstract}
This paper proposes a bimodal biometric system for person identification using two traits, hand geometry and palm texture. The proposed system use complete hand images to find hand geometry and palm texture features. Unlike other multimodal biometric systems, the user does not have to undergo the inconvenience of using two different sensors as two biometrics can be taken from the same image. Palm texture is presented using transform features and hand geometry features are represented as distances between different boundary points. The final decision is made by fusion at decision level in which feature vector are created independently for query image and then compared with the enrollment templates which are stored during database preparation for each biometric trait. This system is tested on the database collected at our institute for 100 people. The Genuine Acceptance Rate(GAR) of the system for fusion is found to be $99.5 \%$. Rotation of hand by 10 degrees gives \%GAR 98.5\%. Equal Error Rate(EER) achieved is 1.11.
\end{abstract}

\section{General Terms}

Biometrics, Pattern Recognition

\section{Keywords}

Biometric Identification, Feature Fusion, Hand Geometry, Multimodal Biometric, Palmprint Identification

\section{INTRODUCTION}

Biometrics, as the science of measuring and compiling distinguishing physical or biological features about an individual, such as facial structure, fingerprints or iris, is a solution for the growing need of security [1][2]. The performance of a biometric system is largely affected by the reliability of the sensor used and the degrees of freedom offered by the features extracted from the sensed signal. This problem can be alleviated by installing multiple sensors that capture different biometric traits. Such systems, known as multimodal biometric systems [3]. Multi-modal biometric system is relatively new application of information fusion while individual biometrics has been used for fairly long time.

In multi-modal biometric system, there are generally four sorts of fusion level [4]:

1. Fusion at sensor level, where images are fused directly or using some transform technique and features are then extracted from fused image.
2. Fusion at feature-extraction level, where the features extracted using two or more sensors are concatenated.

3. Fusion at matching-score level, where the matching scores obtained from multiple matchers are combined.

4. Fusion at decision level, where the accept/reject decisions of multiple systems are consolidated.

Most observers accept that fusion produces better results when performed at the feature extraction level rather than at the data matching or decision levels. This is because the data is combined at its most information rich stage and before contaminations from external sources occur. However, feature level fusion is difficult as the relationship between individual biometric feature spaces may not be straightforward, for example, how can data from fingerprints and faces combined?

Biometric systems that use human hand are based either on palm or hand geometry or palm vein characteristics. Palmprint features, which are composed of principal lines, wrinkles, minutiae, delta points etc. and geometry features include area/size of palm, length and width of fingers. To use palm texture as feature transform based approach is generally used. Work that appear in the literature is mostly based on eigen palm[5], Gabor filters[6][7], Fourier Transform[8] and Wavelet[9][10]. The Gabor filter has good performance for feature extraction however the extraction is time consuming and sensitive to non-linear distortions and rotations. Wavelet based method is also time consuming and band selection is critical process. Hand geometry features generally used are finger length and widths, palm length and width, area/size of palm [11]-[13]. In paper [15] person identification based on hand print is presented which combine palm features, fingerprint features and hand geometry features. The hand shape geometry features are used for coarse level identification and palm and finger features coded by wavelet zero crossing is used in fine level identification. They have used database of only 50 person with 5 images for training and 5 images for testing. It achieved recognition rate of $97 \%$ with EER of 1.5. Main problem is less database and using three biometrics recognition rate achieved is less. Paper [16] again gives idea of hand based authentication using same three biometrics. Database used is of 100 person and used 33 hand shape features, 144 palmprint features and few finger minutiaes which increases its computational as well as space complexity. Combined matching by sum rule gives EER of 3.53. Paper [17] \& [18] finds DCT feature for palm and integrate it with hand shape feature has more length of feature used for person recognition.

In all this recent work it is found that some problems still persist. Most of the algorithms are time consuming and use large size of feature vector. There is complexity in feature 
extraction and they are sensitive to nonlinear distortion and rotations. Further feature level fusion is not easy as different biometrics undergoes different kind of feature extraction.

This paper proposes a novel algorithm for feature extraction based on hand geometry and palmprint which requires less space for storage of features as well as less computational complexity. The paper is organized as follows. In Section 2, the overall system architecture along with the preprocessing module is explained. Section 3 describes the features we extract from hand geometry and palm texture using transform. The experimental set-up and results obtained are discussed in section 4. Result analysis and conclusions are given in section 5 .

\section{SYSTEM ARCHITECTURE}

The detailed system block diagram is shown in Fig. 1. The entire system diagram is briefly described as follows: First, the palmprint and hand geometry image acquisition module uses a digital camera to capture the hand images. This provides a simple, low-cost, non contact, comfortable and user-friendly acquisition mechanism. The preprocessing module employs image processing algorithms to separate the region of interest (ROI) of palm from an input image. Next, the feature extraction module extracts the features of hand geometry and palmprint. Finally, recognition module employs a minimum distance classifier according to Euclidean distance metric to recognize the hand pattern by comparing the feature vector with the enrolled data in the database.

\subsection{Preprocessing Module}

For hand geometry preprocessing following steps are used Step 1: Color to gray transformation - Since gray level image is adequate for hand shape segmentation process, we need to convert color image to gray-level image.

Step 2: Noise reduction and gray-to-binary transformationAveraging filter is applied to remove the noise pixels from the gray level image. Furthermore, the gray level image is binarized.

Step 3: Edge smoothing - Because the binarization possibly generates notch edges, as shown in Fig. 2 (a), we have used morphological opening operation to smooth the edges.

Step 4: Boundary extraction by morphology - The boundary of the hand is extracted by first "eroding" output image of step-3 with the structuring element (disk type) and then performing the image difference between output image of step 3 and its erosion ( Fig. 2 (b)).

$$
\beta(A)=A-(A \ominus \mathrm{B})
$$

Where, $\beta(A)=$ Boundary of $A$

$$
B=\text { Structuring element }
$$

By taking negative image of Fig. 2 (b), we have obtained the contour of hand (Fig. 2 (c)).

Step 5: Locating peak and valley points - This step has much importance for the further processing, because the output image from this step has all reference points i.e. fingertips and valley points, which are useful to extract the ROI of palm. To locate the peak and valley points on the hand boundary, the basic steps are as follows.

- Find the lower left most point on the boundary

(Fig. 2

(d)) and consider this point as reference point for further processing.

- Next, trace the boundary of the hand starting from reference point to accumulate all the boundary pixels.
- Find the Euclidean distance of all boundary pixels with respect to the starting reference point and plot peak and valley points on hand boundary as well as on the gray image (Fig. 2 (e) \& (f)).

In palmprint preprocessing first three steps are same as preprocessing steps of the hand geometry. Peak and valley points of the geometry are used for the extraction of palm ROI.

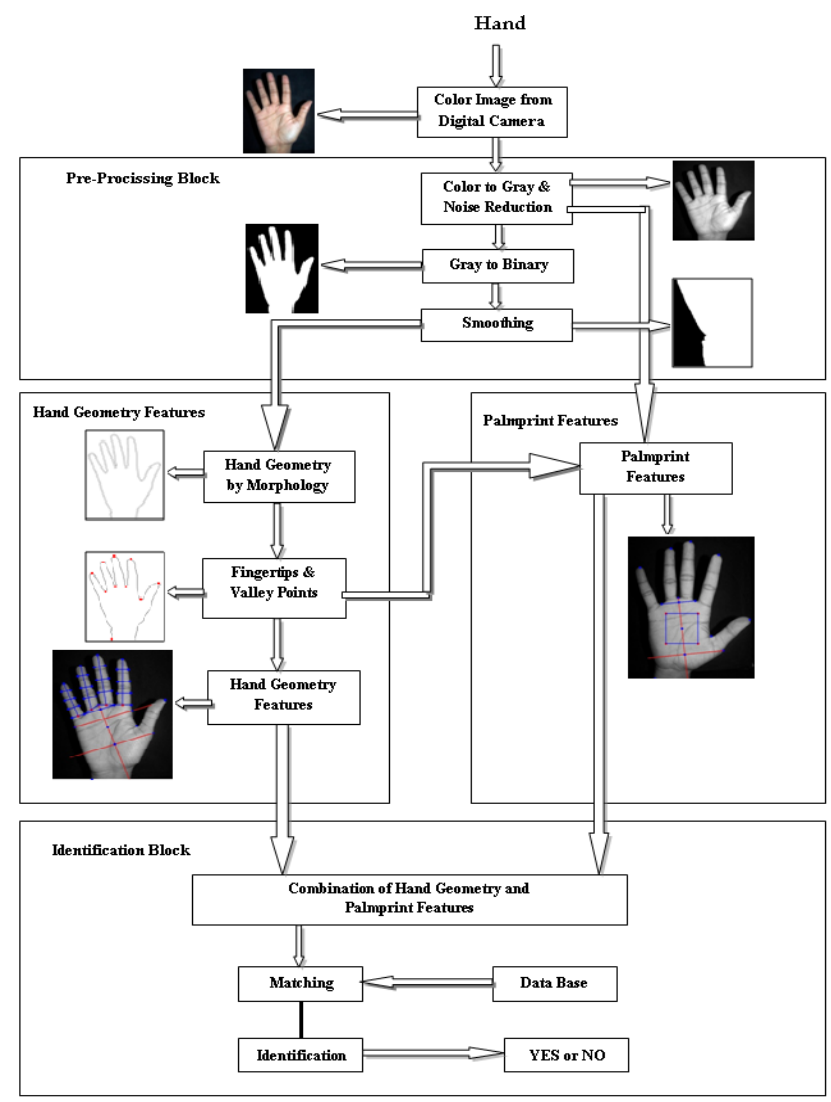

Figure 1: System block diagram

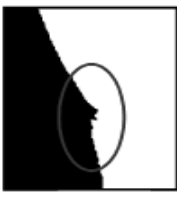

(a)

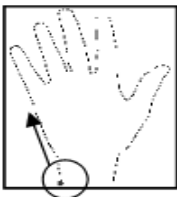

(d)

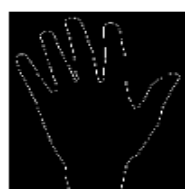

(b)

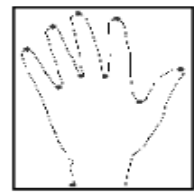

(e)

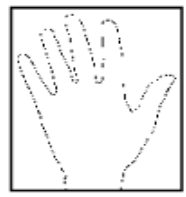

(c)

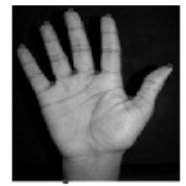

(f)

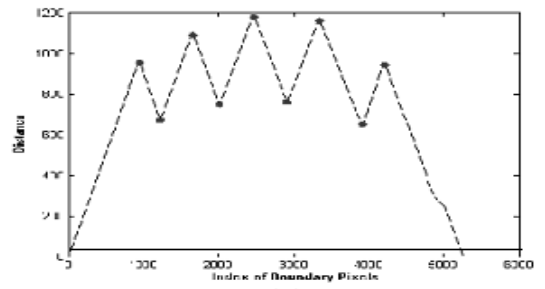

(g)

Figure 2: (a) Notch edges, (b) and (c) boundary of hand, (d) reference point, (e) and (f) location of points on hand contour and gray image, (g) distance plot. 


\section{FEATURE EXTRACTION}

In this module we extract hand geometry features (finger lengths, finger widths, palm width) and palmprint features. All the features of the hand geometry are in the form of the Euclidian distance between two points i.e. between two pixels. The Euclidian Distance between two points $\left.\mathrm{Y}_{1}\right)$ and $\left(\mathrm{X}_{2}, \mathrm{Y}_{2}\right)$ is given by,

$$
E D=\sqrt{\left(X_{1}-X_{2}\right)^{2}+\left(Y_{1}-Y_{2}\right)^{2}}
$$

\subsection{Hand Geometry feature extraction}

Illustrated in Fig. 2, four valley points are located in between the fingers on the hand boundary. Because the middle-finger is the only finger which does not have large spatial variations of its valley points for different placement of the hands, the middle-finger baseline is formed by connecting the second and third (counted from the left) valley points. For remaining finger baseline, we have assumed that two end points of each baseline have the same distance from the respective fingertip point. Using one of the respective valley points as one of the end points we locate the other end point by searching for the point which has the same distance from the fingertip at the another side of the boundary of the finger.

For all fingers, three widths $\left(W_{1}, W_{2}\right.$ and $W_{3}$ in Fig. 3) are taken at different places. These three widths are measured at fixed Euclidian distance of 1/4, 2/4 and 3/4 respectively, with respect to the fingertips. Along with this palm width is also calculated. Thus, total 17 geometrical features (4 finger lengths, 12 finger widths and palm width) are taken into consideration.

\subsection{Palmprint Feature Extraction}

In this section, the cropping of palm ROI and feature extraction using different transforms is explained. Palm ROI cropping is mainly responsible for checking of robustness against rotation and translation of hand images. In [18], full hand image is rotated at particular angle before cropping of palm ROI. They have rotated full hand image such that the algorithm crops the approximate same palm ROI against different rotation of hand. The rotation of complete hand image may take much processing time as we need to project all pixels at particular angle. Instead of rotating full hand image, if somehow, we can crop only interested region of palm at particular angle or at particular slope, then this processing time might get reduced significantly. Such kind of palm ROI cropping is proposed here. The proposed palm ROI cropping is shown in Fig. 4.

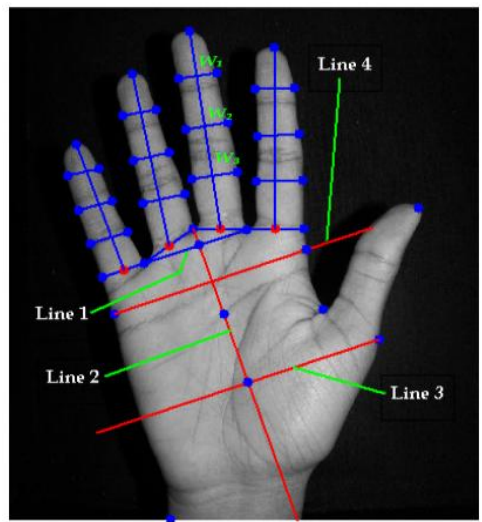

Figure 3: Hand geometry features

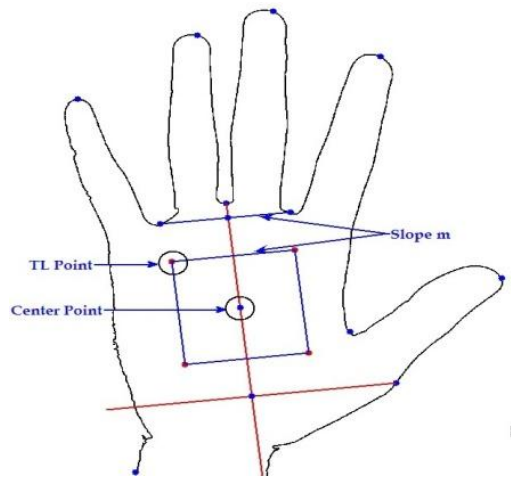

Figure 4: Palm ROI cropping at slope $m$.
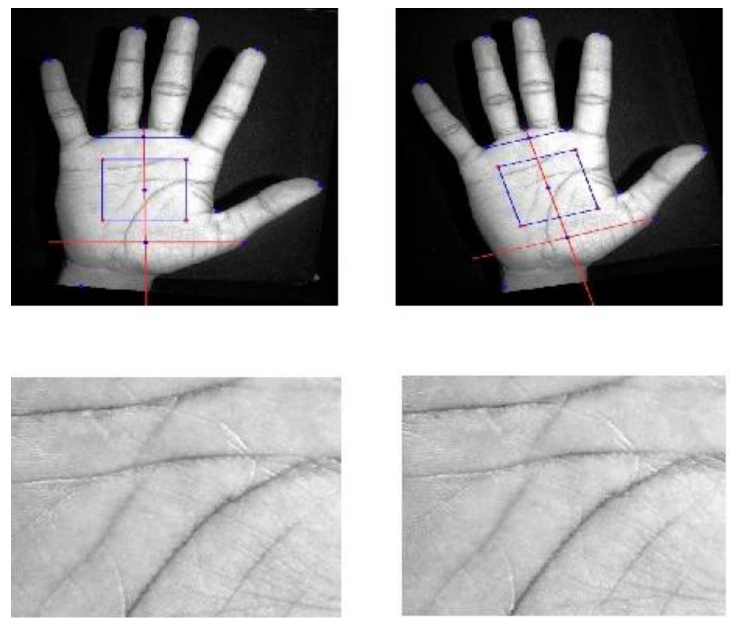

Figure 5: Cropped palm ROI at different rotation angle from same hand image

For palm ROI cropping, the square of particular size $256 \mathrm{x}$ 256 is drawn at Line 1 ( Fig. 3) slope $m$ for extraction of palm ROI. Slope of line between two points $\left(\mathrm{X}_{1}, \mathrm{Y}_{1}\right)$ and $\left(\mathrm{X}_{2}, \mathrm{Y}_{2}\right)$ is given by

$$
m=\frac{\left(y_{1}-y_{2}\right)}{\left(x_{1}-x_{2}\right)}
$$

Here Top Left (TL) point of palm ROI is taken as first reference point to crop the ROI.

The coordinates for the TL point are calculated by

$\mathrm{TL}_{\mathrm{x}}=\left(\right.$ Center $\left._{\mathrm{x}}-128\right)+(128 * \mathrm{~m})$

$\mathrm{TL}_{\mathrm{y}}=\left(\right.$ Center $\left._{\mathrm{y}}-(128 * \mathrm{~m})\right)-128$

Where Center $r_{x}$ and Center $y$ are $\mathrm{x}$ and y coordinate of Center point ( Fig. 4) of palm ROI.

The $\mathrm{x}$ and $\mathrm{y}$ coordinates for the Top Right (TR), Bottom Right (BR) and Bottom Left (BL) point of palm ROI are calculated by

$\mathrm{TR}_{\mathrm{x}}=\mathrm{TL}_{\mathrm{x}}+256$

$\mathrm{TR}_{\mathrm{y}}=\mathrm{TL}_{\mathrm{y}}+(256 * \mathrm{~m})$

$\mathrm{BR}_{\mathrm{x}}=\mathrm{TR}_{\mathrm{x}}-(256 * \mathrm{~m})$

$\mathrm{BR}_{\mathrm{y}}=\mathrm{TR}_{\mathrm{y}}+256$

$\mathrm{BL}_{\mathrm{x}}=\mathrm{BR}_{\mathrm{x}}-256$

$\mathrm{BL}_{\mathrm{y}}=\mathrm{BR}_{\mathrm{y}}-(256 * \mathrm{~m})$

With reference to these four corner points of palm ROI, the palmprint is cropped at slope $m$. Fig. 5 shows the cropped palm ROI from the same hand image at different rotation angles. 
Palmprint feature extraction using different transforms are explained as a further part of this section. How to define the feature is the key point in any biometric identification. A feature with good discriminating ability should exhibit a large variance between individuals and small variance between samples from the same person.

Of the discrete transforms, DCT best approaches the KL and the paper sufficiently utilizes these favorable properties of DCT [14]. The two dimensional DCT of an $\mathrm{M} x \mathrm{~N}$ image $\mathrm{f}(\mathrm{x}$, y) is a separable transform defined as

$T(u, v)$

$=\frac{1}{\sqrt{2 M}} c_{u} c_{v} \sum_{x=0}^{M-1} \sum_{y=0}^{N-1} f(x, y) \cos \left(\frac{(2 x+1) \pi u}{2 M}\right) \cos \left(\frac{(2 y+1) \pi v}{2 N}\right)$ $---(8)$

where $0 \leq \mathrm{x} \leq \mathrm{M}-1,0 \leq \mathrm{y} \leq \mathrm{N}-1$. The DCT is real, orthogonal, fast and separable transform. It has excellent energy compaction for highly correlated data. DCT has become one of the most successful transforms in image processing for the purpose of data compression, feature extraction and recognition.

The two dimensional DFT of an $M x N$ image $f(x, y)$ is a separable transform defined as

$F(u, v)=\frac{1}{M N} \sum_{x=0}^{M-1} \sum_{y=0}^{N-1} f(x, y) e^{-j 2 \pi(u x / M+v y / N)}$ --- (9)

where $0 \leq u \leq M-1, \quad 0 \leq v \leq N-1$. An FFT is an algorithm to compute the same results in only $\mathrm{O}(\mathrm{N} \log \mathrm{N})$ operations. Since the FFT coefficient values contain both real and imaginary parts, we use its absolute value to compute a feature vector. According to characteristic, the DCT and FFT features can be extracted from the palmprint image by considering middle and high frequency bands as shown in Fig. 7(a) and Fig. 7(b).

In this paper region of interest of palm of size $256 \times 256$ is cropped as shown in Fig. 4 and cropped portion of palm is shown in Fig. 6. The size of cropped palm is too big to process hence it is further resized to size of $128 \mathrm{X} 128$ hereafter called as resized ROI. The resized ROI image undergoes no further preprocessing. The algorithm first divides the image into four non overlapping parts around center point as shown in Fig. 6. The 2-D DCT and DFT transforms are applied on each sub-image separately. The transformed coefficients are now grouped into different nine frequency bands (blocks) as shown in Fig. 7. For each numbered block the standard deviation is calculated. Such features are calculated from four sub images and hence form a feature vector of $36(4 \times 9=36)$ which is used in enrollment as well as matching phase. These are stored as float values so take 144 bytes $(36 \times 4)$ to store the feature vector for one person.

The DWT refers to a class of transform that differ not only in transformation kernel employed but also in the fundamental nature of those functions and the way in which they are applied. The image is actually decomposed that is divided into four sub-bands and critically sub sampled by applying DWT as shown in Fig. 8(a). The sub-band LL1 is further decomposed in same manner as shown in Fig. 8(b). Here three level decomposition is carried out. Total 36 features are collected from four sub-images and used as feature vector. In experimentation we have used Daubechies 9 wavelet basis function as it has given better results as compared to other wavelet family.

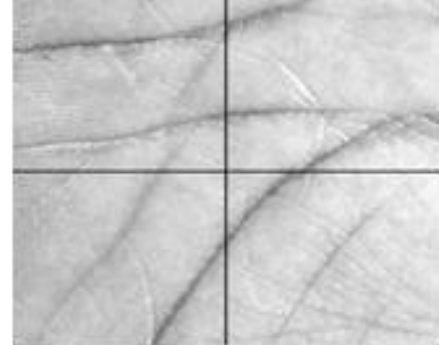

Figure 6. Cropped palm with 4 non overlap region

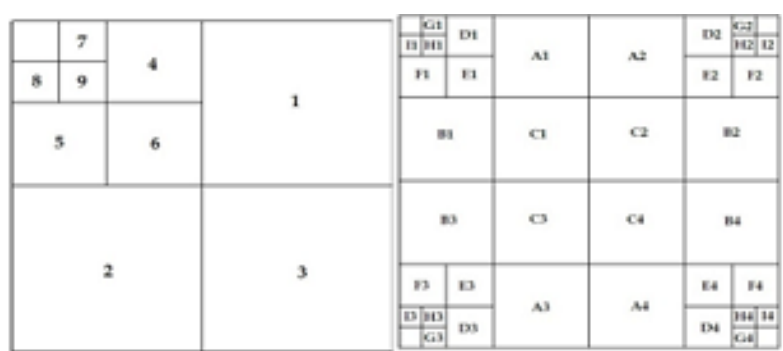

Figure 7. Arrangement of sub-image (a) DCT coefficient (b) DFT coefficient

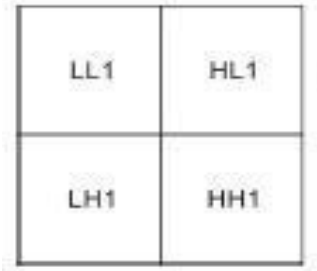

(a) One-Level

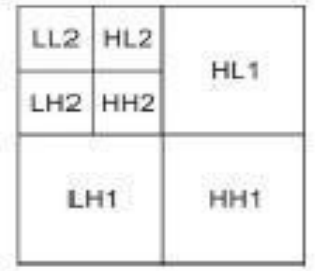

(b) Two-Level
Figure 8. Sub-image DWT decomposition.

\section{Experimental Settings and Results}

Database used consist of 100 person and 8 images of right hand for each person. Volunteers from our institute given their hand image are in the age group of 18 to 50. Here the performance of person identification algorithm based on hand geometry features and transform features from palm are compared using different training and testing set. Thus total 53 (17 hand geometry +36 palm) features are calculated for each of the training set. The database is created using $\mathrm{k}$ hand images where $\mathrm{k}$ varies as 2,4 or 6 . Thus total number of images used for training and testing are given in Table 1. Once feature vector (53) for testing image is calculated it is compared with all database feature vector on one to one basis. Euclidean distance is used to find out the minimum distance match between two dimensional feature vectors of database image and query (test) image. Table 2 shows the $\%$ GAR for individual hand geometry and palmprint.

Table 1. Number of hand images for training and testing

\begin{tabular}{|l|l|l|}
\hline $\mathrm{K}$ & Training Set $(\mathrm{TR})$ & Testing Set $(\mathrm{TS})$ \\
\hline 2 & $2 \times 100=200$ & $6 \times 100=600$ \\
\hline 4 & $4 \times 100=400$ & $4 \times 100=400$ \\
\hline 6 & $6 \times 100=600$ & $2 \times 100=200$ \\
\hline
\end{tabular}


Table 2. \% GAR for individual hand geometry and palmprint

\begin{tabular}{|l|c|l|l|}
\hline Method & $\begin{array}{l}\text { TR=2, } \\
\text { TS=6 }\end{array}$ & $\begin{array}{l}\text { TR=4, } \\
\text { TS=4 }\end{array}$ & $\begin{array}{l}\text { TR=6, } \\
\text { TS=2 }\end{array}$ \\
\hline Hand Geometry & 85.1667 & 81.5000 & 87.5000 \\
\hline Palm (DCT) & 92.3333 & 92.5000 & 97.5000 \\
\hline Palm (DFT) & 93.0000 & 93.7500 & 95.0000 \\
\hline Palm (DWT) & 84.3333 & 86.2500 & 90.5000 \\
\hline
\end{tabular}

Fusion at decision level is done in which separate authentication decision is made for each biometric trait. These decisions are then combined in to a final vote. Table 3 gives \%GAR for combination of hand geometry and palmprint.

Table 3. \% GAR for fusion

\begin{tabular}{|l|c|l|l|}
\hline Method & TR=2, TS=6 & TR=4, TS=4 & TR=6, TS=2 \\
\hline DCT & 96.8333 & 98.0000 & 99.5000 \\
\hline DFT & 97.3333 & 98.0000 & 99.0000 \\
\hline DWT & 94.8333 & 95.2500 & 97.0000 \\
\hline
\end{tabular}

The proposed algorithm is also checked against the different rotation of hand images for fusion with DCT transform. The $\%$ GAR results are shown in table 4. The final identification results are quantified by False Acceptance Rate (FAR) and False Rejection Rate (FRR), which are variable depending on decision threshold (T). Among all transforms, DCT gives better \% GAR, so the distributions of FAR and FRR for different threshold is plotted in Fig. 9 for DCT.

Table 4. \% GAR for different degree of rotation

\begin{tabular}{|l|l|l|l|}
\hline Rotation & $\mathrm{TR}=2, \mathrm{TS}=6$ & $\mathrm{TR}=4, \mathrm{TS}=4$ & $\mathrm{TR}=6, \mathrm{TS}=2$ \\
\hline $2^{\circ}$ & 96.0000 & 96.5000 & 99.0000 \\
\hline $5^{\circ}$ & 96.3333 & 95.5000 & 99.5000 \\
\hline $8^{\circ}$ & 95.0000 & 95.2500 & 98.5000 \\
\hline $10^{\circ}$ & 95.1667 & 94.7500 & 98.5000 \\
\hline
\end{tabular}

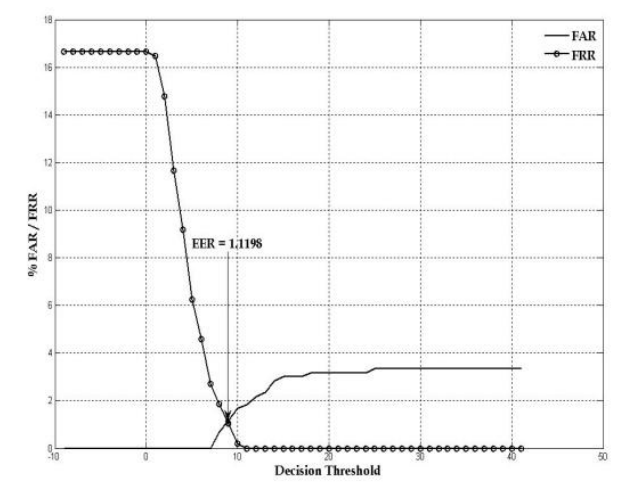

Figure 9. The distribution of $\operatorname{FAR}(T)$ and $\operatorname{FRR}(T)$.

\section{Analysis of Results and Conclusion}

The time required for training and testing equal images (400) at fusion level is around $70 \mathrm{sec}$. Time calculations are done on machine Pentium ${ }^{\circledR}$ D CPU $2.8 \mathrm{GHz}$. Experiments discussed in this paper are implemented using Matlab.
Important feature of the proposed algorithm is that it does not use any kind of preprocessing on extracted ROI of palm and does not use any classification or mapping technique after applying the transform to resized ROI. Further DCT being a real transform requires less computational complexity (compared to DFT or DWT) and can be easily implemented on hardware for real time implementation.

Compared to [15]-[18], number of features used in our method are drastically small. At the same time algorithm presented gives comparable or higher \%GAR. Algorithm is checked in identification mode with simple Euclidean distance measure for 100 person has given less time complexity ( $0.175 \mathrm{sec}$ per hand image). Feature vector is stored as average out values for 2,4 or 6 training images so space requirement is very less $(53 \times 4=212$ bytes). Results shows robustness against translation and rotation.

The objective of this work was to investigate the integration of palmprint transform features and hand geometry features to achieve higher performance that may not be possible with single biometric trait. The individual scores are combined at decision level to develop multimodal biometric system. The performance table and imposter analysis curves shows that multi-modal system performs better as compared to unimodal system achieving EER of 1.1198 and \%GAR more than $99 \%$. Further analysis of three transforms shows that DCT outperforms in terms of recognition rate, computational complexity and time complexity. Also experimental results indicate that the proposed method is robust with respect to translation and rotation upto 10 degrees. More rotation invariance can be achieved, by adaptive cropping of palm ROI according to rotation of hand.

\section{REFERENCES}

A. Jain, R. Bolle and S. Pankanti (eds.). Biometrics: Personal Identification In Networked Society, Boston, Mass: Kluwer Academic Publishers, 1999.

[2] Loris Nanni, Alessandra Lumini. Wavelet Decomposition Tree Selection for Palm and Face Authentication, Pattern Recognition: 343-353, Letters 29, (2008)

[3] Arun Ross, Anil Jain, Jian-Zhong Qian. Information Fusion in Biometrics, proceedings of $3^{\text {rd }}$ Int. Conference on Audio and Video Based Person Authentication:354359, Sweden, 2001.

[4] Slobodan Ribaric, Ivan Fratric. A Biometric Identification System Based on Eigenpalm and Eigenfinger Features, IEEE Transaction on PAMI, vol. 27, No. 11, Nov. 2005.

[5] G. Lu, D. Zhang, and K. Wang. Palmprint Recognition Using Eigenpalms Features, Pattern Recognition Letters, vol. 24, issue 9-10:1463-1467, 2003.

[6] D. Zhang,W. Kong, J. You, and M.Wong. Online palmprint identification, IEEE Transactions on Pattern Analysis and Machine Intelligence, vol. 25, no. 9, pp. 1041-1050, 2003.

[7] Adams Wai-Kin Kong, David Zhang. Competitive Coding Scheme for Palmprint Verification, Proceedings of the 17th International Conference on Pattern Recognition (ICPR'04)

[8] W. Li, D. Zhang, and Z. Xu. Palmprint Identification by Fourier Transform, International Journal of Pattern 
Recognition and Artificial Intelligence, vol.16, no. 4, pp. 417-432, 2002.

[9] Lei Zhang and David Zhang. Characterization of Palmprints by Wavelet Signatures via Directional Context Modeling, IEEE Transaction on SMC-B, Vol. 34, No. 3: 1335-1347, June 2004.

[10] K Y Edward Wong, G. Sainarayanan, Ali Chekima. Palmprint Identification Using Wavelet Energy, Proceedings of Int.Conf. on Intelligent and Advanced Systems, Malaysia, 2007.

[11] Hui Yan, Duo Long, A novel bimodal identification approach based on hand-print, IEEE 2008 Congress on Image and Signal Processing, pp. 506-510.

[12] Fan Yang, Baofeng Ma, Didi Yao, Chenyan Fang, Shundong Zhao and Xiangmin Zhou, Information Fusion of Biometrics Based-on Fingerprint, Hand Geometry and Palm-print, IEEE Workshop on Automatic Identification Advanced Technologies, June 2007.

[13] Ajay Kumar, David Zhang, Combing Fingerprint, Palmprint and Hand-Shape For User Authentication,
Proceedings of $18^{\text {th }}$ Int. Conf. on Pattern Recognition (ICPR) 2006, vol. 4: 549-552.

[14] A. K. Jain, Fundamentals of Digital Image Processing. Englewood Cliffs, NJ: Prentice-Hall, 1989.

[15] Hui Yan, Duo Long, A novel bimodal identification approach on hand print, 2008 Congress on Image and Signal Processing.

[16] Ajay Kumar, David Zhang, Combining Fingerprint, Palmprint and Hand shape for User Authentication, $18^{\text {th }}$ International Conference on Pattern Recognition (ICPR'06)

[17] Ajay Kumar, David Zhang, Integrating Shape and Texture for Hand Verification, ICIG04

[18] Ajay Kumar and David Zhang, Personal Recognition Using Hand Shape and Texture, IEEE Transactions on Image Processing, Vol. 15, No. 8 , pp. $2454 \quad-\quad 2461, \quad$ August 2006. 